\title{
Spectral-free methods in the theory of hereditarily indecomposable Banach spaces
}

\author{
N. de Rancourt
}

\begin{abstract}
We give new and simple proofs of some classical properties of hereditarily indecomposable Banach spaces, including the result by W. T. Gowers and B. Maurey that a hereditarily indecomposable Banach space cannot be isomorphic to a proper subspace of itself. These proofs do not make use of spectral theory and therefore, they work in real spaces as well as in complex spaces. We use our method to prove some new results. For example, we give a quantitative version of the latter result by Gowers and Maurey and deduce that Banach spaces that are isometric to all of their subspaces should have an unconditional basis with unconditional constant arbitrarily close to 1 . We also study the homotopy relation between into isomorphisms in hereditarily indecomposable spaces.
\end{abstract}

\section{Introduction}

In this paper, unless otherwise specified, when speaking about a Banach space (or simply a space), we shall mean an infinite-dimensional Banach space, and by subspace of a Banach space, we shall always mean infinite-dimensional, closed subspace. By operator, we shall always mean bounded linear operator, and by isometry, we shall mean linear (non-necessarily sujective) isometry.

In 1993, W. T. Gowers and B. Maurey [5] built the first example of a Banach space containing no unconditional basic sequence, thus solving the longstanding unconditional basic sequence problem. The space they built actually has a much stronger property: it is hereditarily indecomposable (HI), i.e. no two subspaces of it are in topological direct sum. In the same paper, the authors prove several properties of H] spaces. Among them, the following:

Theorem 1.1. An HI space is isomorphic to no proper subspace of itself.

Mathematics Subject Classification (2010): 46B03, 46B04, 46B20, 47A53

The author was partially supported by the joint FWF-GAČR Grant No. 17-33849L: Filters, Ultrafilters and Connections with Forcing

Keywords: Hereditarily indecomposable Banach spaces, Fredholm theory 
Following Maurey [8], an operator $T: X \rightarrow Y$ between two Banach spaces will be called an into isomorphism if it is an isomorphism between $X$ and its image (or equivalently, if there exists $c>0$ such that $\forall x \in X\|T(x)\| \geqslant c\|x\|$ ). Recall that an operator $S: X \rightarrow Y$ between two Banach spaces is strictly singular if no restriction of $S$ to a subspace of $X$ is an into isomorphism. In their paper, Gowers and Maurey get Theorem 1.1 for complex $\mathrm{HI}$ spaces as an immediate consequence (using basic Fredholm theory) of the following result:

Theorem 1.2. If $X$ is a complex $[\mathrm{H}]$ space then every operator $T: X \rightarrow X$ can be written $T=\lambda \operatorname{Id}_{X}+S$, where $\lambda \in \mathbb{C}$ and $S$ is a strictly singular operator.

Their proof of Theorem 1.2 makes use of Fredholm theory and spectral theory, thus explaining the fact that it only works for complex spaces. Actually, it turns out that this result is not true in general for real $\overline{\mathrm{HI}}$ spaces. For such spaces $X$, the authors prove a general structural result for operators from $X$ to $X$, similar to Theorem 1.2 , by passing to the complexification of $X$. From this result, they deduce Theorem 1.1 for real HI spaces. As they mention, they do not know any direct proof of Theorem 1.1 in the real case. Later, V. Ferenczi [2] gave a new proof of Theorem 1.2 using no spectral theory but rather Banach algebra methods (he actually proves a more general result); however, as far as the author of the present article knows, no direct proof of Theorem 1.1 for real spaces has been known by now.

The main aim of this paper is to introduce new methods in the theory of [H] spaces, using no spectral theory but only Fredholm theory, and thus working in real $\mathrm{HI}$ spaces as well as in complex $[\mathrm{HI}$ spaces. These methods will allow us to give new and simpler proofs of some classical properties of $\mathrm{HI}$ spaces, including Theorem 1.1, this will be done in section 3. Our hope is that these new methods can be adapted more easily to broader contexts. We present such an adaptation in section 4, where a quantitative version of Theorem 1.1 is proved. This allows us to start an investigation of the isometrically homogeneous space problem, asking if a space that is isometric to all of its subspaces should necessarily be isometric to $\ell_{2}$. Finally, in section 5 , we prove some results about the homotopy relation between into isomorphisms from $\mathrm{HI}$ spaces. We deduce from them that the general linear group of the real $[\mathrm{HI}$ space built by Gowers and Maurey in [5] has exactly 4 connected components.

We start this paper with recalling some results in Fredholm theory, in section 2 ,

\section{Fredholm theory}

In this section, we recall some results in Fredholm theory that will be useful in the rest of this paper. For a more detailed presentation and proofs of these results, we refer to the survey [8] on Banach spaces with few operators, that contains a good introduction to Fredholm theory (see sections 3, 4 and 6).

In the rest of this paper, all Banach spaces will be over the field $\mathbb{K}:=\mathbb{R}$ or $\mathbb{C}$, the proofs working as well in both cases. 
Let $X, Y$ be two Banach spaces. Let $\mathcal{L}(X, Y)$ denote the space of bounded operators from $X$ to $Y$ (when $X=Y$, then this space will simply be denoted by $\mathcal{L}(X)$ ). Equip $\mathcal{L}(X, Y)$ with the operator norm denoted by $\|\cdot\|$, and with the associated topology. For $T \in \mathcal{L}(X, Y)$, let $\mathrm{n}(T) \in \mathbb{N} \cup\{+\infty\}$ and $\mathrm{d}(T) \in \mathbb{N} \cup\{+\infty\}$ denote respectively the dimension of the kernel of $T$ and the codimension of the image of $T$. The operator $T$ is said to be semi-Fredholm if it has closed image and if one of the numbers $\mathrm{n}(T)$ and $\mathrm{d}(T)$ is finite, and Fredholm if both numbers $\mathrm{n}(T)$ and $\mathrm{d}(T)$ are finite (this implies that $T$ has closed image). Let $\mathcal{F} r e d(X, Y)$ and $\hat{\mathcal{F}} r e d(X, Y)$ denote respectively the set of Fredholm operators and of semi-Fredholm operators from $X$ to $Y$, seen as subsets of $\mathcal{L}(X, Y)$ with the induced topology. For $T \in \hat{\mathcal{F}} \operatorname{red}(X, Y)$, the Fredholm index of $T$ is defined by $\operatorname{ind}(T)=\mathrm{n}(T)-\mathrm{d}(T) \in \mathbb{Z} \cup\{-\infty,+\infty\}$. One of the fundamental results of Fredholm theory is the following:

Theorem 2.1. The Fredholm index ind : $\hat{\mathcal{F}} \operatorname{red}(X, Y) \longrightarrow \mathbb{Z} \cup\{-\infty,+\infty\}$ is locally constant.

Say that an operator $T: X \rightarrow Y$ is finitely singular if there exists a finite-codimensional subspace $X_{0}$ of $X$ such that $T_{\uparrow X_{0}}$ is an into isomorphism. An operator which is not finitely singular will be called infinitely singular. We have the two following results:

Proposition 2.2. An operator $T: X \rightarrow Y$ is finitely singular if and only if it is semiFredholm with $\operatorname{ind}(T)<+\infty$.

Proposition 2.3. An operator $T: X \rightarrow Y$ is infinitely singular if and only if for every $\varepsilon>0$, there exists a subspace $Y$ of $X$ such that $\left\|T_{\uparrow Y}\right\| \leqslant \varepsilon$.

Finally we mention a last result that we will not use in our proofs, but which is the key of the originial proof of Theorem 1.1 from Theorem 1.2.

Proposition 2.4. Let $T: X \rightarrow Y$ be a Fredholm operator and $S: X \rightarrow Y$ be a strictly singular operator. Then $T+S$ is Fredholm and $\operatorname{ind}(T+S)=\operatorname{ind}(T)$.

The latter result, combined with Theorem 1.2, gives that every element of $\mathcal{L}(X)$, for $X$ a complex $\mathrm{HI}$ space, is either strictly singular or Fredholm with index 0. In particular, such an operator cannot be an isomorphism between $X$ and a proper subspace.

\section{Subspaces, quotients and operators}

In this section we give a new proof of Theorem 1.1 and of some related results. Some elements of our proofs were already present in [5]; to make this paper self-contained, we shall state them as lemmas and prove them.

Given $X$ a Banach space and $Y \subseteq X$ a subspace, the inclusion embedding $Y \hookrightarrow X$ will be denoted by $\mathrm{i}_{Y, X}$ in the rest of this paper. We say that $\lambda \in \mathbb{K}$ is an infinitely singular value of the operator $T: Y \rightarrow X$ when $T-\lambda \mathrm{i}_{Y, X}$ is infinitely singular. The next lemma was already present as a remark in [5]. 
Lemma 3.1. If $X$ is a Banach space, $Y \subseteq X$ an $[H 1$ subspace, and $T: Y \rightarrow X$ an operator, then $T$ has at most one infinitely singular value.

Proof. Suppose that $\lambda, \mu \in \mathbb{K}$ are two infinitely singular values of $T$. Let $\varepsilon>0$; by Proposition 2.3 we can find two subspaces $U_{\varepsilon}, V_{\varepsilon}$ of $Y$ such that $\left\|\left(T-\lambda \mathrm{i}_{Y, X}\right)_{\mid U_{\varepsilon}}\right\| \leqslant \varepsilon$ and $\left\|\left(T-\mu \mathrm{i}_{Y, X}\right)_{\mid V_{\varepsilon}}\right\| \leqslant \varepsilon$. The subspaces $U_{\varepsilon}$ and $V_{\varepsilon}$ are not in topological direct sum, so we can find $u_{\varepsilon} \in S_{U_{\varepsilon}}$ and $v_{\varepsilon} \in S_{V_{\varepsilon}}$ such that $\left\|u_{\varepsilon}-v_{\varepsilon}\right\| \leqslant \varepsilon$. And we have:

$$
\begin{aligned}
|\lambda-\mu| & =\left\|\lambda u_{\varepsilon}-\mu u_{\varepsilon}\right\| \\
& \leqslant\left\|\lambda u_{\varepsilon}-\mu v_{\varepsilon}\right\|+|\mu| \cdot\left\|v_{\varepsilon}-u_{\varepsilon}\right\| \\
& \leqslant\left\|\lambda u_{\varepsilon}-T\left(u_{\varepsilon}\right)\right\|+\left\|T\left(u_{\varepsilon}\right)-T\left(v_{\varepsilon}\right)\right\|+\left\|T\left(v_{\varepsilon}\right)-\mu v_{\varepsilon}\right\|+|\mu| \varepsilon \\
& \leqslant \varepsilon+\|T\| \cdot\left\|u_{\varepsilon}-v_{\varepsilon}\right\|+\varepsilon+|\mu| \varepsilon \\
& \leqslant(2+|\mu|+\|T\|) \varepsilon .
\end{aligned}
$$

Thus, making $\varepsilon$ tend to 0 , we deduce that $\lambda=\mu$.

The next lemma is the key of our proof. It is valid for every Banach space $X$, not necessarily $\mathrm{HI}$,

Lemma 3.2. Let $X$ be a Banach space and $T \in \mathcal{L}(X)$ be a semi-Fredholm operator with nonzero index. Then $T$ has at least two distinct real infinitely singular values, a positive one and a negative one.

Proof. For $t \in[0,1]$, define $T_{t}=t T+(1-t) \operatorname{Id}_{X}$. We show that there exists $t \in(0,1)$ such that $T_{t}$ is infinitely singular; this will imply that $\frac{t-1}{t}$ is a negative infinitely singular value of $T$. Suppose not. Then by Proposition 2.2, for every $t \in[0,1], T_{t}$ is semi-Fredholm. So letting $f(t)=\operatorname{ind}\left(T_{t}\right)$, we define a function $f:[0,1] \longrightarrow \mathbb{Z} \cup\{-\infty,+\infty\}$; by Theorem 2.1. this function is locally constant, so constant. This is a contradiction since $f(0)=0$ and $f(1) \neq 0$.

We prove in the same way that $T$ has a positive infinitely singular value, considering the operators $T_{t}^{\prime}=t T-(1-t) \operatorname{Id}_{X}$.

We can now conclude the proof of Theorem 1.1. Let $X$ be an $\mathrm{HI}$ space and $T \in \mathcal{L}(X)$ be an isomorphism from $X$ to a proper subspace of $X$. Then $T$ is semi-Fredholm with $\operatorname{ind}(T)<0$, so by Lemma 3.2 it has at least two distinct infinitely singular values. This contradicts Lemma 3.1 .

We now give a new proof of a generalization of Theorem 1.1 first proved by Ferenczi [3].

Theorem 3.3. Let $X$ be an $[H]$ space, and let $Z \subseteq Y$ be subspaces of $X$, with $Z$ not necessarily infinite-dimensional. Suppose that either the inclusion $Y \subseteq X$ is strict, or $Z \neq\{0\}$. Then $X$ is not isomorphic to $Y / Z$.

Proof. We follow the same approach as for Theorem 1.1. Let $\pi: Y \rightarrow Y / Z$ be the quotient map. Suppose that there exists an isomorphism $T: Y / Z \rightarrow X$. Then both 
$\mathrm{i}_{Y, X}$ and $T \circ \pi$ are semi-Fredholm operators $Y \rightarrow X$, and we have ind $\left(\mathrm{i}_{Y, X}\right) \leqslant 0$ and $\operatorname{ind}(T \circ \pi) \geqslant 0$. Moreover, one of the latter inequalities has to be strict. So ind $\left(\mathrm{i}_{Y, X}\right) \neq$ $\operatorname{ind}(T \circ \pi)$.

By the continuity of the Fredholm index, there should exist $t \in(0,1)$ such that $t T \circ \pi+(1-t) \mathrm{i}_{Y, X}$ is not semi-Fredholm. This means that there exists $\lambda<0$ such that $T \circ \pi-\lambda \mathrm{i}_{Y, X}$ is not semi-Fredholm, so infinitely singular by Proposition 2.2. In the same way, considering the operators $t T \circ \pi-(1-t) \mathrm{i}_{Y, X}$, we see that there is a $\mu>0$ such that $T \circ \pi-\mu \mathrm{i}_{Y, X}$ is infinitely singular. This contradicts Lemma 3.1 .

We finish this section with a direct proof of the following result, which, as said before, is an immediate consequence of Theorem 1.2 and Proposition 2.4 in the special case of complex spaces, but which is also valid for real spaces.

Theorem 3.4. Let $T \in \mathcal{L}(X)$, where $X$ is an H1 space. Then either $T$ is Fredholm with index 0 , or $T$ is strictly singular.

In order to prove Theorem 3.4 , we need the following lemma, that was already present as a remark in [5].

Lemma 3.5. Let $T \in \mathcal{L}(X)$, where $X$ is an $[\mathrm{HI}$ space. Then $T$ is infinitely singular if and only if it is strictly singular.

Proof. One implication directly follows from the definitions. For the other, suppose that $T$ is infinitely singular. We fix $Y$ a subspace of $X$ and $\varepsilon>0$, and we want to find $y \in S_{Y}$ such that $\|T(y)\|<\varepsilon$.

Since $T$ is infinitely singular, there exists a subspace $Z$ of $X$ such that $\left\|T_{\uparrow Z}\right\| \leqslant \frac{\varepsilon}{2}$. Since $Y$ and $Z$ are not in topological direct sum, we can find $y \in S_{Y}$ and $z \in S_{Z}$ with $\|y-z\| \leqslant \frac{\varepsilon}{2\|T\|}$. Then we have:

$$
\|T(y)\| \leqslant\|T(z)\|+\|T(y-z)\| \leqslant \frac{\varepsilon}{2}+\|T\| \cdot \frac{\varepsilon}{2\|T\|} \leqslant \varepsilon,
$$

as wanted.

Proof of Theorem 3.4. Suppose that $T$ is not strictly singular. By Lemma 3.5, it is finitely singular, so by Proposition 2.2, it is semi-Fredholm. If it does not have index 0, then by Lemma 3.2, it has at least two infinitely singular values, contradicting Lemma 3.1 


\section{A quantitative version}

Say that a Banach space $X$ is homogeneous is it is isomorphic to all of its subspaces, and isometrically homogeneous if it is isometric to all of its subspaces. In [1, the authors ask the following question:

Question 4.1 (Cúth-Doležal-Doucha-Kurka). Is every isometrically homogeneous Banach space isometric to $\ell_{2}$ ?

This problem, that we will call the isometrically homogeneous space problem, is an isometric version of the homogeneous space problem, asking whether every homogeneous space is isomorphic to $\ell_{2}$. The latter problem was solved positively in the nineties as a combination of the three followings results:

- every Banach space either has a subspace isomorphic to $\ell_{2}$, or has a subspace without an unconditional basis (Komorowski-Tomczak-Jaegermann, [7);

- every Banach space either has a subspace with an unconditional basis, or has an HI subspace (Gowers' first dichotomy, [4]);

- and Theorem 1.1, by Gowers and Maurey.

To investigate the isometrically homogeneous space problem, it is tempting to try to find quantitative versions of the results above. Gowers' first dichotomy admits a quantitative version, that will be presented below (Theorem 4.5) and which was proved by Gowers himself as a step of the proof of the same non-quantitative result. In this section, we give a quantitative version of Theorem 1.1. This version, combined with the quantitative version of Gowers' first dichotomy, will give us the following result:

Theorem 4.2. Let $X$ be an isometrically homogeneous Banach space. Then for every $\varepsilon>0, X$ admits a $(1+\varepsilon)$-unconditional basis.

We don't know if there is a sufficiently good quantitative version of KomorowskiTomczak-Jaegermann's theorem to solve positively the isometrically homogeneous space problem.

In the rest of this section, we fix a Banach space $X$. Observe that if $Y$ and $Z$ are two subspaces of $X$, then $Y$ and $Z$ are not in topological direct sum if and only if for every $C \geqslant 1$, there exists $y \in Y$ and $z \in Z$ with $\|y+z\|>C\|y-z\|$. This motivates the following definition:

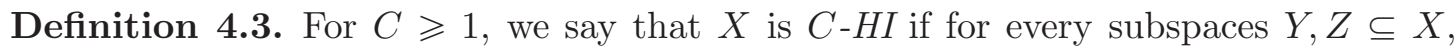
there exists $y \in Y$ and $z \in Z$ such that $\|y+z\|>C\|y-z\|$.

In particular, $X$ is $\mathrm{HI}$ if and only if it is $C$-HI for arbitrarily large $C$. A slightly different definition of $C$ [HI spaces had already been given by Gowers in [4, for spaces with a basis, only taking into account block-subspaces. However, is is easy to see that these two notions are equivalent up to a constant. 
Recall that two Banach spaces $Y$ and $Z$ are said to be $C$-isomorphic, for some $C \geqslant 1$, if there is an isomorphism $T: Y \rightarrow Z$ with $\|T\| \cdot\left\|T^{-1}\right\| \leqslant C$. In particular, two spaces are 1-isomorphic if and only if they are isometric. Our quantitative version of Theorem 1.1 is the following:

Theorem 4.4. Let $C \geqslant 1$. Suppose that $X$ is $(C+\varepsilon) H 1$ for some $\varepsilon>0$. Then $X$ is $C$-isomorphic to no proper subspace of itself.

Proof. Suppose that $X$ is $C$-isomorphic to a proper subspace $Y \subseteq X$. Let $T: X \rightarrow Y$ be an isomorphism with $\|T\| \leqslant C$ and $\left\|T^{-1}\right\|=1$; we see $T$ as an operator $X \rightarrow X$. By Lemma 3.2, $T$ has at least two real infinitely singular values, a positive one that we denote by $\lambda$ and a negative one that we denote by $-\mu$. Replacing $T$ with $-T$ if necessary, we can assume that $\lambda \leqslant \mu$. We let $\nu=\mu-\lambda$. For every $a<1$ and every $x \in X$, we have $\|T(x)-a x\| \geqslant\|T(x)\|-a\|x\| \geqslant(1-a)\|x\|$, so $T-a \operatorname{Id}_{X}$ is an into isomorphism and in particular, it is finitely singluar. We deduce that $\lambda \geqslant 1$.

We now use a similar method as in the proof of Lemma 3.1 to conclude. Fix $\varepsilon>0$. Then there exists subspaces $Y, Z \subseteq X$ such that $\left\|\left(T-\lambda \operatorname{Id}_{X}\right)_{\uparrow Y}\right\| \leqslant \varepsilon$ and $\left\|\left(T+\mu \operatorname{Id}_{X}\right)_{\mid Z}\right\| \leqslant \varepsilon$. Thus, for every $y \in Y$ and $z \in Z$, we have:

$$
\begin{aligned}
(\lambda+\mu)\|y+z\| & =\|2 \lambda y+2 \mu z+(\mu-\lambda)(y-z)\| \\
& \leqslant 2\|\lambda y+\mu z\|+\nu\|y-z\| \\
& \leqslant 2(\|\lambda y-T(y)\|+\|T(y)-T(z)\|+\|T(z)+\mu z\|)+\nu\|y-z\| \\
& \leqslant 2 \varepsilon(\|y\|+\|z\|)+2 C\|y-z\|+\nu\|y-z\| \\
& \leqslant 2 \varepsilon(\|y+z\|+\|y-z\|)+(2 C+\nu)\|y-z\|
\end{aligned}
$$

Since $\lambda \geqslant 1$, we have $2+\nu \leqslant \lambda+\mu$. Using this inequality on the left-hand side and the fact that $2 C+\nu \leqslant(2+\nu) C$ on the right-hand side, we get that:

$$
(2+\nu-2 \varepsilon)\|y+z\| \leqslant((2+\nu) C+2 \varepsilon)\|y-z\| .
$$

We deduce that $X$ is not $\frac{(2+\nu) C+2 \varepsilon}{2+\nu-2 \varepsilon}$-HI. Since $\frac{(2+\nu) C+2 \varepsilon}{2+\nu-2 \varepsilon}$ tends to $C$ when $\varepsilon$ tends to 0 , we get the wanted result.

Now the quantitative version of Gowers' first dichotomy, proved in [4] (see Corollary $3.2)$ is the following:

Theorem 4.5 (Gowers). Let $C \geqslant 1$ and $\varepsilon>0$. Then either $X$ has a subspace with $a$ $(C+\varepsilon)$-unconditional basis, or a $C-H]$ subspace.

This, combined with Theorem 4.4, is enough to prove Theorem 4.2. Indeed, if $X$ is isometrically homogeneous, then, fixing $\varepsilon>0$ and applying Theorem 4.4 to $C=1$, we get that $X$ is not $(1+\varepsilon)-[\mathrm{HI}$, so contains no $(1+\varepsilon)-[\mathrm{HI}$ subspace. By Theorem 4.5 applied to $C=1+\varepsilon$, we get that $X$ has a subspace with a $(1+2 \varepsilon)$-unconditional basis, so $X$ itself has a $(1+2 \varepsilon)$-unconditional basis. 


\section{Homotopy between into isomorphisms}

Given $X$ and $Y$ two Banach spaces, let $\operatorname{Emb}(Y, X)$ the set all $T \in \mathcal{L}(Y, X)$ that are into isomorphisms. This is an open subset of $\mathcal{L}(Y, X)$. In the case where $X=Y$, this set is the general linear group of $X$ nd will be denoted as $\operatorname{GL}(X)$. Given two operators $S, T \in \operatorname{Emb}(Y, X)$, we say that $Y$ and $X$ are homotopic if there is a continuous mapping:

$$
\begin{aligned}
{[0,1] } & \rightarrow \operatorname{Emb}(Y, X) \\
t & \mapsto T_{t}
\end{aligned}
$$

such that $T_{0}=S$ and $T_{1}=T$. Homotopy is an equivalence relation whose classes are exactly the connected components of $\operatorname{Emb}(Y, X)$. In [8], the following result is mentionned as an exercise, in the special case of complex spaces:

Theorem 5.1. Let $X$ be an HI spaces and $Y, Z$ to subspaces of $X$ that are isomorphic. Then there is an into isomorphism $T: Y \rightarrow X$ with $T(Y)=Z$, and such that $T$ is homotopic to $\mathrm{i}_{Y, X}$ in $\operatorname{Emb}(Y, X)$.

In this section, we give a proof of Theorem 5.1 working as well in the complex as in the real case. This result can be seen as an analogue of Theorem 1.1 for subspaces: if two subspaces of an $\mathrm{HI}$ space are isomorphic, then none of them can be "too deep" compared to the other. In particular, Theorem 1.1 is a consequence of Theorem 5.1; given $X$ an $\left[\mathrm{HI}\right.$ space and $Y \subseteq X$ a proper subspace, if $Y$ is isomorphic to $X$, then $\operatorname{Id}_{X}$ should be homotopic to an isomorphism from $X$ to $Y$, which is impossible by continuity of the Fredholm index.

We actually prove a slightly stronger result than Theorem 5.1. Given $Y$ a Banach space, recall that an operator $R: Y \rightarrow Y$ is a reflection of $Y$ if there exists $H \subseteq Y$ a hyperplane and $x_{0} \in Y \backslash\{0\}$ such that $\forall x \in H R(x)=x$ and $R\left(x_{0}\right)=-x_{0}$. Call an antireflection of $Y$ an operator of the form $-R$, where $R$ is a reflection of $Y$. Any two reflections $R_{0}$ and $R_{1}$ of the same space $Y$ are homotopic: indeed, given $x_{0}, x_{1} \in Y \backslash\{0\}$ and $l_{0}, l_{1} \in Y^{*} \backslash\{0\}$ such that for all $i, \operatorname{ker}\left(l_{i}\right)=\operatorname{ker}\left(R_{i}-\operatorname{Id}_{Y}\right)$ and $R_{i}\left(x_{i}\right)=-x_{i}$, then, it is not hard to find a continuous path between $\left(x_{0}, l_{0}\right)$ and $\left(\delta x_{1}, \varepsilon l_{1}\right)$ for some choice of signs $\delta, \epsilon \in\{-1,1\}$ in the set $\left\{(x, l) \in X \times X^{*} \mid l(x) \neq 0\right\}$. This directly gives an homotopy between $R_{0}$ and $R_{1}$. In the same way, any two antireflections of the same space are homotopic. In what follows, for simplicity of notation, given $Y \subseteq X$ two Banach spaces, we will sometimes confound an automorphism $U$ of $Y$ with the into isomorphism $\mathrm{i}_{Y, X} \circ U: Y \rightarrow X$. The theorem we prove is the following:

Theorem 5.2. Let $X$ be a Banach space, $Y$ be an $[H]$ subspace of $X$, and $T: Y \rightarrow X$ be an into isomorphism. Then $T$ is either homotopic, in $\operatorname{Emb}(Y, X)$, to $\mathrm{i}_{Y, X}$, or to $-\mathrm{i}_{Y, X}$, or to all reflections of $Y$, or to all antireflections of $Y$. Moreover, this result can be refined in the following cases:

1. If $Y \neq X$, then $T$ is either homotopic to $\mathrm{i}_{Y, X}$, or to $-\mathrm{i}_{Y, X}$;

2. If $\mathbb{K}=\mathbb{C}$, then $T$ is homotopic to $\mathrm{i}_{Y, X}$. 
Theorem 5.2 implies Theorem 5.1, indeed, if $U$ is any isomorphism $Y \rightarrow Z$, then Theorem 5.2 shows that $U$ is homotopic, in $\operatorname{Emb}(Y, X)$, to an automorphism $V$ of $Y$, so $U \circ V^{-1}$ is an isomorphism $Y \rightarrow Z$ which is homotopic, in $\operatorname{Emb}(Y, X)$, to $\mathrm{i}_{Y, X}$. Another consequence of Theorem 5.2 is that if $Y$ is any $\mathrm{HI}$ space and $X$ any Banach space, then $\operatorname{Emb}(Y, X)$ has:

- at most four connected components if $\mathbb{K}=\mathbb{R}$ and $X$ is isomorphic to $Y$;

- at most two connected components if $\mathbb{K}=\mathbb{R}$ and $X$ is not isomorphic to $Y$;

- at most one connected component if $\mathbb{K}=\mathbb{C}$.

In what follows, we use the following notation. If $Y$ and $Z$ are (finite- or infinitedimensional) subspaces of the same space $X$ that are in topological direct sum, and if $T: Y \rightarrow X$ and $U: Z \rightarrow X$ are two operators, then we denote by $T \oplus U$ the unique operator $Y \oplus Z \rightarrow X$ extending both $T$ and $U$. The main ingredient in the proof of Theorem 5.2 is the following lemma:

Lemma 5.3. Let $X$ be a Banach space, $Z \subseteq X$ be a subspace, and $F \subseteq X$ be a finitedimensional subspace such that $Z \cap F=\{0\}$. Let $Y=Z \oplus F$, let $T: Y \rightarrow X$ be an into isomorphism, and suppose that $T_{\uparrow Z}$ is homotopic to $\mathrm{i}_{Z, X}$ in $\operatorname{Emb}(Z, X)$. Let $R$ be a reflection of $F$. Then, in $\operatorname{Emb}(Y, X), T$ is either homotopic to $\mathrm{i}_{Y, X}$, or to $\mathrm{i}_{Z, X} \oplus R$.

Proof of Lemma 5.3. We first prove that the special case where $\operatorname{dim}(F)=1$ implies the general case. This will be done by induction on $\operatorname{dim}(F)$. Suppose $\operatorname{dim}(F) \geqslant 2$ and let $R$ be a reflection of $F$. Let $G=\operatorname{ker}\left(R-\operatorname{Id}_{F}\right)$ and let $x_{0} \in F \backslash\{0\}$ such that $R\left(x_{0}\right)=-x_{0}$, so we have $F=G \oplus \mathbb{K} x_{0}$. Let $R^{\prime}$ be any reflection of $G$ and let $R^{\prime \prime}=R^{\prime} \oplus \mathrm{i}_{\mathbb{K} x_{0}, F}$, which is a reflection of $F$. Applying the induction hypothesis to $Z, G$, the operator $T_{\uparrow Z \oplus G}$ and the reflection $R^{\prime}$, we obtain that $T_{\uparrow Z \oplus G}$ is either homotopic to $\mathrm{i}_{Z \oplus G, X}$, or to $\mathrm{i}_{Z, X} \oplus R^{\prime}$. So either $T_{\uparrow Z \oplus G}$, or $T_{\uparrow Z} \oplus\left(T_{\uparrow G} \circ R^{\prime}\right)$ is homotopic to $\mathrm{i}_{Z \oplus G, X}$. Applying the one-dimensional case to $Z \oplus G, \mathbb{K} x_{0}$, respectively the operator $T_{\uparrow Y}$ or the operator $T_{\uparrow Z} \oplus\left(T_{\uparrow F} \circ R^{\prime \prime}\right)$, and the reflection $-\operatorname{Id}_{\mathbb{K} x_{0}}$, we get that one of the operators $T_{\uparrow Y}$ and $T_{\uparrow Z} \oplus\left(T_{\uparrow F} \circ R^{\prime \prime}\right)$ is homotopic to one of the operators $\mathrm{i}_{Y, X}$ and $\mathrm{i}_{Y \oplus G, X} \oplus\left(-\mathrm{i}_{\mathbb{K} x_{0}, X}\right)=$ $\mathrm{i}_{Z, X} \oplus R$. Summarizing, we get that the operator $T_{\uparrow Y}$ is homotopic to one of the four following operators:

- $\mathrm{i}_{Y, X}$;

- $\mathrm{i}_{Z, X} \oplus R$;

- $\mathrm{i}_{Z, X} \oplus R^{\prime \prime}$;

- $\mathrm{i}_{Z, X} \oplus\left(R \circ R^{\prime \prime}\right)$.

But since the reflections $R$ and $R^{\prime \prime}$ are homotopic in $\operatorname{Emb}(F, F)$, we are actually in one of the first two cases, which finishes the first part of this proof.

We now prove the result in the special case where $\operatorname{dim}(F)=1$. Let $\left(\widetilde{T}_{t}\right)_{t \in[0,1]}$ be an homotopy between $\mathrm{i}_{Z, X}$ and $T_{\uparrow Z}$ in $\operatorname{Emb}(Z, X)$. By local constancy of the Fredholm 
index, for every $t \in[0,1]$ we have $\widetilde{T}_{t}(Z) \neq X$. So by a standard compacity argument, we can find $0=t_{0}<t_{1}<\ldots<t_{n}=1$ and vectors $x_{1}, \ldots, x_{n} \in X$ such that for every $i<n$ and every $t \in\left[t_{i}, t_{i+1}\right]$, we have $x_{i} \notin \widetilde{T}_{t}(Z)$. We can even assume that $F=\mathbb{K} x_{0}$ and that $x_{n-1}=T\left(x_{0}\right)$.

For every $t \in[0,1]$ and every $x \in X$, we abusively denote by $\widetilde{T}_{t} \oplus x$ the unique operator $U: Y \rightarrow X$ such that $U_{\uparrow Z}=\widetilde{T}_{t}$ and $U\left(x_{0}\right)=x$. Since $x_{0} \mapsto-x_{0}$ is the only reflection of $F$, what we have to prove is that $T=\widetilde{T}_{1} \oplus x_{n-1}$ is homotopic to $\widetilde{T}_{0} \oplus \varepsilon x_{0}$ in $\operatorname{Emb}(Y, X)$ for some sign $\varepsilon \in\{-1,1\}$. To prove that, first observe that for every $i<n$ and for every $\varepsilon \in\{-1,1\}, \widetilde{T}_{t_{i}} \oplus \varepsilon x_{i}$ and $\widetilde{T}_{t_{i+1}} \oplus \varepsilon x_{i}$ are homotopic in $\operatorname{Emb}(Y, X)$. So to conclude, it is enough to show that for every $1 \leqslant i<n$ and for every sign $\varepsilon \in\{-1,1\}$, there exists a $\operatorname{sign} \delta \in\{-1,1\}$ such that $\widetilde{T}_{t_{i}} \oplus \varepsilon x_{i}$ is homotopic to $\widetilde{T}_{t_{i}} \oplus \delta x_{i-1}$ in $\operatorname{Emb}(Y, X)$.

To see this this, suppose that both segments $\left[\varepsilon x_{i}, x_{i-1}\right]$ and $\left[\varepsilon x_{i},-x_{i-1}\right]$ intersect $\widetilde{T}_{t_{i}}(Z)$. This means that there exists $t, t^{\prime} \in(0,1)$ such that $t \varepsilon x_{i}+(1-t) x_{i-1} \in \widetilde{T}_{t_{i}}(Z)$ and $t^{\prime} \varepsilon x_{i}-\left(1-t^{\prime}\right) x_{i-1} \in \widetilde{T}_{t_{i}}(Z)$. So $\left(\frac{t}{1-t}+\frac{t^{\prime}}{1-t^{\prime}}\right) \varepsilon x_{i} \in \widetilde{T}_{t_{i}}(Z)$, a contradiction. Thus, there exists $\delta \in\{-1,1\}$ such that the segment $\left[\varepsilon x_{i}, \delta x_{i-1}\right]$ does not intersect $\widetilde{T}_{t_{i}}(Z)$. This means that the family $\left(\widetilde{T}_{t_{i}} \oplus\left(t \varepsilon x_{i}+(1-t) \delta x_{i-1}\right)\right)$ is an homotopy between $\widetilde{T}_{t_{i}} \oplus \varepsilon x_{i}$ and $\widetilde{T}_{t_{i}} \oplus \delta x_{i-1}$ in $\operatorname{Emb}(Y, X)$, concluding the proof.

Proof of Theorem 5.2. We first prove the general case. Lemma 3.1 show us that $T$ has at most one infinitely singular value. Replacing $T$ with $-T$ if necessary, we may assume that $T$ has no nonpositive infinitely singular value. This implies that for every $t \in[0,1]$, the operator $t T+(1-t) \mathrm{i}_{Y, X}$ is finitely singular. For every $t \in[0,1]$, we can find $Z_{t}$ a finite-codimensional subspace of $Y$ such that $\left.\left(t T+(1-t) \mathrm{i}_{Y, X}\right)\right|_{Z_{t}}$ is an into isomorphism; there exists $U_{t}$ a neighborhood of $t$ in $[0,1]$ such that for every $t^{\prime} \in U_{t}$, $\left.\left(t^{\prime} T+\left(1-t^{\prime}\right) i_{Y, X}\right)\right|_{Z}$ is still an into isomorphism. Select $t_{1}, \ldots t_{n} \in[0,1]$ such that $\bigcup_{i=1}^{n} U_{t_{i}}=[0,1]$, and let $Z=\bigcap_{i=1}^{n} Z_{t_{i}}$. Then $Z$ is a finite-codimensional subspace of $Y$ such that for every $t \in[0,1],\left(t T+(1-t) i_{Y, X}\right)_{\uparrow Z}$ is an into isomorphism. Thus, $T_{\uparrow Z}$ and $\mathrm{i}_{Z, X}$ are homotopic in $\operatorname{Emb}(Z, X)$.

Taking $F$ any complement of $Z$ in $Y$ and $R$ any reflection of $Z$, we can apply Lemma 5.3 to $Z, F, T$ and $R$, showing that $T$ is either homotopic to $\mathrm{i}_{Y, X}$, or to $\mathrm{i}_{Z, X} \oplus R$ in $\operatorname{Emb}(Y, X)$. This concludes the general case, since $\mathrm{i}_{Z, X} \oplus R$ is a reflection of $Y$.

To prove the case where $Y \neq X$, it is enough to show that any reflection of $Y$ is homotopic to $\mathrm{i}_{Y, X}$ in $\operatorname{Emb}(Y, X)$. Given $R$ such a reflection, consider $x_{0} \notin Y$ any vector, let $Z=Y \oplus \mathbb{K} x_{0}$ and $R^{\prime}=R \oplus \mathrm{i}_{\mathbb{K} x_{0}, Z}$. This defines a reflection of $Z$. This reflection is homotopic to the reflection $\mathrm{i}_{Y, Z} \oplus\left(-\mathrm{i}_{\mathbb{K} x_{0}, Z}\right)$ of $Z$ in $\operatorname{Emb}(Z, X)$, and this homotopy restricts into an homotopy between $R$ and $\mathrm{i}_{Y, X}$ is $\operatorname{Emb}(Y, X)$.

To prove the case where $\mathbb{K}=\mathbb{C}$, it is enough to prove that for any complex space $X$, and for any decomposition $X=Y \oplus Z$ (where $Y$ and $Z$ can be finite- or infinitedimensional), the operator $\mathrm{i}_{Y, X} \oplus\left(-\mathrm{i}_{Z, X}\right)$ is homotopic to $\operatorname{Id}_{X}$. An homotopy is given by $\left(\mathrm{i}_{Y, X} \oplus\left(e^{i \pi t} \mathrm{i}_{Z, X}\right)\right)_{t \in[0,1]}$. 
Remark 5.4. Theorem 5.2 is optimal, in the sense that there exists a real $[\mathrm{HI}$ space $X$ such that for every reflection $R$ of $X$, the operators $\operatorname{Id}_{X},-\operatorname{Id}_{x}, R$ and $-R$ are pairwise non-homotopic in $\operatorname{Emb}(X, X)$, and for every subspace $Y$ of $X$, the operators $i_{Y, X}$ and $-\mathrm{i}_{Y, X}$ are non-homotopic in $\operatorname{Emb}(Y, X)$. In particular, there exists a real Banach space whose general linear group has exactly four connected components. We sketch the proof of these facts below.

Take for $X$ the real space built by Gowers and Maurey in [5]. It has the following property: for every subspace $Y$ of $X$, every operator $T: Y \rightarrow X$ has the form $\lambda \operatorname{Id}+S$, where $\lambda \in \mathbb{R}$ and $S$ is strictly singular. It is not hard to see that $\lambda$ is continuous in $T$ (use for example a similar method as in the proof of Lemma 3.1), thus showing that $\mathrm{i}_{Y, X}$ and $-\mathrm{i}_{Y, X}$ cannot be homotopic in $\operatorname{Emb}(Y, X)$.

We now prove that $\operatorname{Id}_{X}$ cannot be homotopic to a reflection of $X$ in $\operatorname{Emb}(X, X)$. For a real space Banach $Y$ and an operator $T: Y \rightarrow Y$, we denote its complexification by $T^{\mathbb{C}}: Y^{\mathbb{C}} \rightarrow Y^{\mathbb{C}}$. Denote by $\sigma(T)$ the spectrum of an operator $T$ on a complex space. Recall the following facts:

1. If $T$ is an operator on a real space, then the spectrum of $T_{\mathbb{C}}$ is invariant under conjugation, and if $\lambda$ is an eigenvalue of $T_{\mathbb{C}}$, then $\bar{\lambda}$ is also one, with the same multiplicity;

2. The spectrum of a strictly singular operator on a complex space consists in 0 together with a either finitely many nonzero eigenvalues with finite multiplicity, or a sequence of nonzero eigenvalues with finite multiplicity converging to 0 (see 8 , Proposition 6.1);

3. If $T: Y \rightarrow Y$ is an operator on a complex space, and $V$ an open subset of $\mathbb{C}$ such that $\sigma(T) \cap V$ consists in finitely many eigenvalues of $T$ with finite multiplicity, then for every $S \in \mathcal{L}(Y)$ close enough to $T, \sigma(S) \cap V$ consists in finitely many eigenvalues of $S$ and the sum of their multiplicities is equal to the sum of the multiplicities of the eigenvalues of $T$ in $V$ (see [6], Chapter Four, subsection 3.5).

Now if there exists an homotopy $\left(T_{t}\right)_{t \in[0,1]}$ in $\operatorname{Emb}(X, X)$ between $T_{0}=\operatorname{Id}_{X}$ and $T_{1}=R$ a reflection, then without loss of generality we can assume that for every $t \in[0,1]$, $T_{t}=\operatorname{Id}_{X}+S_{t}$, where $S_{t}$ is strictly singular. In particular, $0 \notin \sigma\left(T_{t}^{\mathbb{C}}\right), \sigma\left(T_{t}^{\mathbb{C}}\right)$ contains only finitely many negative elements and all of these elements are eigenvalues with finite multiplicity. Now if $t \in[0,1]$ and $V$ is an open subset of $\mathbb{C}$ such that $V \cap \mathbb{R}=(-\infty, 0)$ and such that $\sigma\left(T_{t}^{\mathbb{C}}\right) \cap V$ is exactly the set of negative eigenvalues of $T_{t}^{\mathbb{C}}$, then for $s$ in a neigborhood of $t$, the sum of the multiplicities of the eigenvalues of $T_{s}^{\mathbb{C}}$ that are in $V$ is equal to the sum of the multiplicities of the negative eigenvalues of $T_{t}^{\mathbb{C}}$. By invariance of the spectrum under conjugation, the sum of the multiplicities of $T_{s}^{\mathbb{C}}$ that are in $V \backslash(0, \infty)$ is even. Thus, the parity of the sum of the multiplicities of the negative eigenvalues of $T_{t}^{\mathbb{C}}$ is localy constant, so constant. This contradicts the fact that this sum is 0 for $T_{0}$, and 1 for $T_{1}$. 


\section{References}

[1] M. Cúth, M. Doležal, M. Doucha, and O. Kurka. Polish spaces of Banach spaces. Complexity of isometry classes and generic properties. arXiv:1912.03994.

[2] V. Ferenczi. Operators on subspaces of hereditarily indecomposable Banach spaces. Bull. London Math. Soc., 29:338-344, 1997.

[3] V. Ferenczi. A Banach space dichotomy theorem for quotients of subspaces. Studia Math., 180:111-131, 2007.

[4] W. T. Gowers. An infinite Ramsey theorem and some Banach-space dichotomies. Ann. Math., 156:797-833, 2002.

[5] W. T. Gowers and B. Maurey. The unconditional basic sequence problem. J. Amer. Math. Soc., 6:851-874, 1993.

[6] T. Kato. Perturbation theory for linear operators. Classics in mathematics. Springer, 1980.

[7] R. A. Komorowski and N. Tomczak-Jaegermann. Banach spaces without local unconditional structure. Israel J. Math., 89:205-226, 1995.

[8] B. Maurey. Operator theory and exotic Banach spaces. 1996. Notes available on the author's webpage.

N. de Rancourt, Department of Logic, Faculty of Arts, Charles University, nám. Jana Palacha 2, 11638 Praha 1, CZECH REPUBlic

Current adress: Universität Wien, Institut für Mathematik, Kurt Gödel Research Center, Augasse 2-6, UZA 1 - Building 2, 1090 Wien, AUSTRIA

Email address: noe.de.rancourt@univie.ac.at 